\title{
Modeling Geometric Varieties with Given Differential Characteristics and Its Application
}

\author{
Evgeniy Konopatskiy ${ }^{[0000-0003-4798-7458]}$ and Andrey Bezditnyi ${ }^{2^{[0000-0003-0528-9731]}}$ and \\ Oksana Shevchuk ${ }^{10000-0002-9224-0671]}$ \\ ${ }^{1}$ Donbas National Academy of Civil Engineering and Architecture, Ukraine \\ ${ }^{2}$ Sevastopol branch of «Plekhanov Russian University of Economics», Russian Federation \\ e.v.konopatskiy@mail.ru, pereverten_1985@mail.ru, \\ o.a.shevchukedonnasa.ru
}

\begin{abstract}
The article describes an approach to modeling geometric manifolds with specified differential properties, which is based on the use of geometric interpolants of a multidimensional space. A geometric interpolant is understood as a geometric object of a multidimensional space passing through predetermined points in advance, the coordinates of which correspond to the initial experimental and statistical information. Principles for determining geometric interpolants and an example of an analytical description of a 3-parameter geometric interpolant belonging to a 4-dimensional space in the form of a geometric scheme and a computational algorithm based on a sequence of point equations are given. The main direction of practical use of geometric interpolants is geometric modeling of multifactor processes and phenomena, but they can also be an effective tool for multivariate approximation. Based on this, the article presents a general approach to modeling geometric manifolds with given differential properties and its application in the form of a numerical solution of differential equations by approximating it using geometric interpolants of a multidimensional space. To implement an approach to modeling geometric manifolds with specified differential properties, it is proposed to use a computational algorithm consisting of 10 points. The advantages of using geometric interpolants for the numerical solution of differential equations are highlighted.
\end{abstract}

Keywords: Geometric Varieties, Differential Characteristics, Geometrical Interpolant.

\section{Introduction}

Differential characteristics of geometric manifolds have important theoretical and applied significance. The need to study them in parallel with the development of mathematical analysis led to the emergence of differential geometry as a separate branch of mathematics.

Copyright (C) 2020 for this paper by its authors. Use permitted under Creative Commons License Attribution 4.0 International (CC BY 4.0). 
In differential geometry, the geometric meaning of differentiation and integration operations is considered. So, the geometric meaning of the first derivative of the function is reduced to determining the tangent to the curve, and the second derivative - to determining the curvature of the curve. The study of differential characteristics often led to the creation of new geometric objects based on their prototypes. For example, an evolute, that has found wide application in engineering practice, is a geometric place of points (a curve) that are the centers of curvature of the original curve. Another example of using differential characteristics of curved lines is the work [1-3], in which the author proposed the use of functional curves for geometric modeling of physical processes in the form of a set of functionally interconnected lines of trajectory, speed and acceleration. This approach is valid for defining other geometric manifolds: compartments of surfaces, hypersurfaces, etc., which leads to differentiation of the function of many variables. For example, the point equation of the torso surface as a geometric place of tangents to its return edge is nothing more than a differential equation of a function of two variables. If we consider the analytical description of geometric manifolds obtained by differential-geometric transformations, then in their structure they are nothing more than a certain differential equation and are its geometric equivalent. Then, between differential equations and geometric manifolds with given differential characteristics, there is a mutually inverse logical relationship. That is, geometrical manifolds with given differential characteristics can serve as a particular solution of differential equations in the same way as differential equations are used to analytically describe geometric manifolds with given differential characteristics.

Differential equations can have both exact and approximate (numerical) solutions. In engineering practice, there are often differential equations whose solution cannot be expressed in elementary mathematical functions. Therefore, numerical methods based on multidimensional interpolation and approximation are used for their particular solution. An example is the iso-geometric method for solving partial differential equations [4-5], which is based on the concept that the same set of functions is used both for an analytical description of an approximating geometric object and for approximating a solution. In the works of Sophus Li [6-7], a detailed study of point groups of plane transformations is proposed, whereas differential equations are found not as the main object of research, but as an auxiliary apparatus. Another approach based on modeling geometric manifolds with pre-defined properties is proposed in [8-10]. The main concept of the proposed approach to the numerical solution of differential equations [8-10] is to use geometric interpolants of a multidimensional space [11] to model geometric manifolds with specified differential characteristics.

\section{Principles for determining geometric interpolants in a multidimensional space}

In General, a geometric interpolant is a geometric object of a multidimensional affine space that passes through pre-defined points whose coordinates correspond to the original experimental and statistical information [11]. The process of determining geometric interpolants consists of two principal stages: building a tree of a geometric model 
and its analytical description using point equations [12] and computational algorithms based on them. The tree of a geometric model is a geometric scheme for the graphical construction of a geometric interpolant based on the method of a moving simplex [12] using as formative elements algebraic curves passing through pre-defined points obtained on the basis of Bernstein polynomials [13].

1 the principle. From simple to complex. Any, even the most complex geometric interpolant, is represented by a set of simpler geometric interpolants, united with each other by a generating line. For example, a geometric interpolant of 2-dimensional space, which is a section of the response surface, can be represented as a set of 1-dimensional geometric interpolants (reference lines passing through pre-defined points), connected to each other using a generating line. The more we set the current parameters that determine the geometric interpolant, the more complicated will be the structure of the tree of the geometric model and, accordingly, the computational algorithm for constructing it. However, it still consists of simpler interconnected elements.

2 the principle. Belonging of one geometric object to another. The geometric theory of multidimensional interpolation [11] is based on the simplest principle of belonging of one geometric object to another accepted in descriptive geometry, according to which a straight line belongs to a plane if two points of this line belong to a plane. In turn, a point belongs to a plane if it belongs to a straight line lying in that plane. Considering a straight line as a special case of a curve, and a plane as a special case of a surface, we obtain the statement, that is necessary for defining a 2-parameter geometric interpolant in the form of a compartment of a surface, that passes through predetermined points and belongs to 3-dimensional space.

Statement 1. In order for the source points to belong to a 2-parameter geometric interpolant, you must organize them as reference lines (in descriptive geometry, the term guide lines is used), and then combine them using a generating line that passes through the current points of the reference line family.

Then all points, that belong to the reference lines will belong to the desired 2-parameter interpolant. It should be noted that the formative element of both the reference and forming lines of the geometric interpolant are arcs of algebraic curves passing through pre-set points. Only when forming reference lines, the curves pass through the source points (whose coordinates correspond to the original experimental and statistical information), and when forming the generating line-through the current points of reference lines of the geometric interpolant.

Generalizing the proposed approach to defining geometric interpolants on a 4-dimensional space, we obtain a statement akin to statement 1.

Statement 2. In order for the source points to belong to a 3-parameter geometric interpolant, you must organize the source points as 2-parameter geometric interpolants (a family of reference surfaces), united in a hypersurface by a forming line passing through the current points of the family of reference surfaces.

Similarly, you can define an $n$-parametric geometric interpolant, that belongs to an $(n+1)$-dimensional space.

As an example, consider the tree of a geometric model of a 3-parameter geometric interpolant (Fig. 1). 


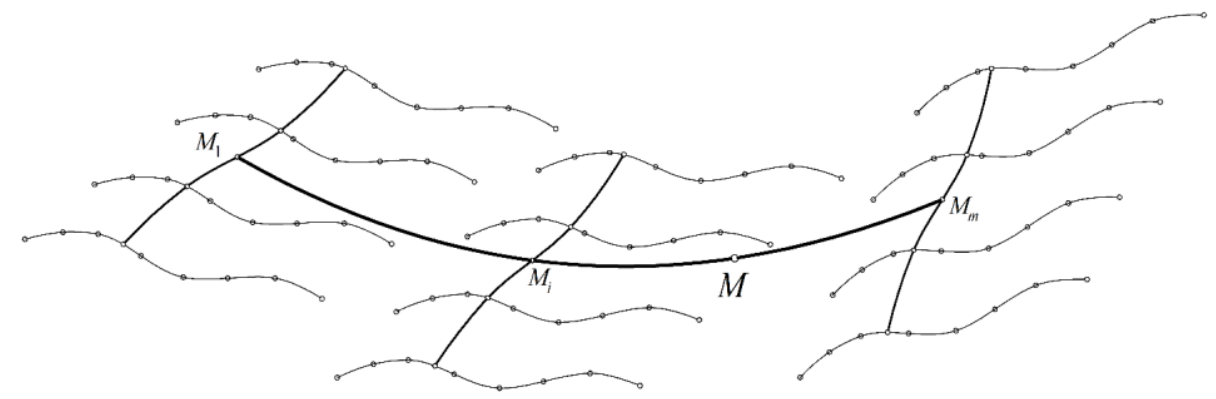

Fig. 1. Tree of the geometric model of the 3-parametric geometric interpolant

An analytical description of a 3-parametric geometric interpolant in the form of a 3parametric hypersurface is represented by the following sequence of point equations:

$$
\left[\begin{array}{l}
M_{i j}=\sum_{k=1}^{l} M_{i j k} p_{i j k}(u) \\
\ldots \ldots \ldots \ldots \ldots \ldots \ldots \ldots \ldots \ldots \ldots \ldots \ldots \ldots \ldots \ldots \ldots \ldots \ldots \ldots \ldots \ldots \ldots \ldots \\
M_{i}=\sum_{j=1}^{n} M_{i j} q_{i j}(v) \\
\ldots \ldots \ldots \ldots \ldots \ldots \ldots \\
M=\sum_{i=1}^{m} M_{i} r_{i}(w)
\end{array}\right.
$$

where $M$ - the current point of the hypersurface compartment that passes through the pre-set points in the number of $m\ulcorner n\ulcorner l$;

$M_{i}$ - current point of the generator $i$ a section of the surface that passes through pre-defined points, which is a reference for building a hypersurface;

$M_{i j}$ - current point $j$ the reference arc of a curve that passes through preset points;

$M_{i j k}$ - the starting points through which the desired hypersurface compartment should pass. The coordinates of these points correspond to the original experimental and statistical data;

$p_{i j k}(u)$ - functions from the parameter that define the type of reference lines;

$q_{i j}(v)$ - functions from the parameter $v$, defining the type of forming lines compartments surfaces;

$r_{i}(w)$ - functions from the parameter $w$, defining the form of the hypersurface compartment forming line; $l$ - number of source points in each reference line;

$n$ - the number of control lines to build the reference surfaces;

$m$ - number of reference surfaces for building a hypersurface;

$i$ - serial number of the reference surface; 
$j$ - sequential number of the reference line;

$k$ - the sequential number of the starting point in each reference line;

$u, v$ and $w$-current parameters that change from 0 to 1 .

To move from point equations to parametric ones, you need to perform a coordinate calculation, which is the analytical equivalent of projecting a 3-parameter hypersurface on the axis of the global coordinate system.

The main direction of practical use of the geometric theory of multidimensional interpolation is geometric modeling of multi-factor processes and phenomena [14]. In addition, it can be effectively used in geometric computer modeling as a forming tool for modeling geometric shapes and bodies passing through pre-set points. However, another effective application of the geometric theory of multidimensional interpolation is possible. In particular, in [8-10, 15-16], 3 variants of using geometric interpolants for multidimensional approximation are proposed and studied:

- by selecting nodal points from the original set of points [15].

- by minimizing the sum of lengths between nodes and source points [16-17].

- by setting the required differential characteristics at the nodal points [8-10].

The second variant [16-17] is presented as a result of generalization of the least squares method to a multidimensional space using its geometric interpretation. In [810], an additional field of application of the third variant of multidimensional approximation is proposed: numerical solution of differential equations using geometric interpolants, which is based on the modeling of geometric manifolds with specified differential characteristics.

\section{General approach to modeling geometric manifolds with specified differential characteristics}

The main idea of the proposed approach to modeling geometric manifolds with given differential properties is as follows: in multidimensional nodes interpolation network of points the condition of the original differential equation is satisfied, which provides the required differential properties of the simulated geometric object. Multidimensional interpolation is performed automatically between nodes in the point network by using geometric interpolants. This is the numerical solution of the original differential equation.

To implement an approach to modeling geometric manifolds with specified differential characteristics, we suggest using the following algorithm:

1. We should to form a network of points of the necessary dimension and density depending on original differential equation. We recommend using a regular point network, but if necessary, you can also use an irregular point network.

2. Structure the generated network of points to build a tree of the geometric model.

3. Use formative arcs of algebraic curves passing through the front set points, for the analytical description of the geometric interpolant. In this case, we form a computational algorithm equivalent to the tree of the geometric model. 
4. Perform a coordinate calculation and, if using a regular network of points, go from the system of parametric equations for the analytical description of the geometric interpolant to its explicit equation. If an irregular network of points is used, additional differentiation of parametric equations by parameters is necessary, followed by solving a homogeneous system of first-degree differential equations by the Kramer method [18].

5. Enter the coordinates of the points corresponding to the initial and boundary conditions of the original differential equation. Geometric representation of initial and boundary conditions can be geometric objects: points, lines, surfaces, etc., that pass through pre-defined points.

6. Differentiate the resulting equations and substitute them into the original differential equation. Thus, we obtain an equation of an approximating geometric interpolant with pre-defined differential characteristics.

7. Substitute parameter values at the nodal points, thereby forming local system of linear algebraic equations (SLAE).

8. In the case of using piecewise polynomial approximation, repeat the first 7 points of the computational algorithm several times. With this, we accumulate local SLAEs to form a global SLAE. In the case of using a continuous geometric interpolant, this point of the algorithm must be ignored and go to the next.

9. Solve the resulting SLAE and determine the necessary parameter values at the nodal points of the geometric interpolant. Then insert the calculation result into the equation of the approximating geometric interpolant from the 6th point.

10. Analyze the result and check its validity. If the results are not accurate enough, increase the number of nodal points in the geometric interpolant.

It should be noted that the order of the initial differential equation is directly related to the order of the shape-forming curves passing through the predetermined points of the geometric interpolant. Moreover, to ensure the correct result, it is necessary that the order of the forming curves passing through the predetermined points is higher than the order of the original differential equation.

\section{Practical application of the proposed approach}

The main practical application of the proposed approach to modeling geometric manifolds with specified differential characteristics is the numerical solution of differential equations in computer-aided design and calculation systems by approximating their solutions using geometric interpolants of a multidimensional space. At the same time, it should be noted that the proposed approach is sufficiently universal and that it is possible to increase both the number of variables of the original differential equation and its order. Moreover, if the solution can be represented with sufficient accuracy as a smooth function, it is preferable to use continuous geometric interpolants. In other cases, it is possible to use piecewise polynomial functions in the form of multidimensional contours using algebraic curves of different smoothness order.

The proposed approach can be effectively used primarily for the numerical solution of partial differential equations for which the solution is not expressed in elementary 
functions. In [9], an example of a numerical solution of an inhomogeneous heat equation using a 16-point geometric interpolant is given, and the results of the numerical solution are compared with the reference solution obtained by the method of separating variables. At the same time, further use of the resulting numerical solution in the form of a polynomial dependence is more preferable for engineering calculations, in comparison with the equation obtained by the method of dividing variables.

It should be noted, that by covering a multidimensional network of source points with a single geometric interpolant, a finite super element for approximating differential equations is formed, which has the following advantages:

1. Curved geometric objects of a multidimensional affine space are simultaneous carriers of several points, which significantly reduces the "piecewise" of the final function and the number of necessary calculations.

2. With regard to solving problems of strength and stability, the final super element can be used on any network of points, while maintaining a curved component, which ensures that geometric nonlinearity is taken into account when solving problems of strength and stability. At the same time, the possibility of geometric modeling of physical, structural, genetic and other non-linearity remains.

3. Due to the invariant properties of the parameter of point equations, there is no need to use guide cosines.

4. Using the geometric theory of multidimensional interpolation, it is possible to generalize the approach to modeling geometric manifolds with given differential characteristics both in the direction of increasing the number of variables and in the direction of increasing the order of derivatives of the original differential equation. Moreover, the number of variables is limited only by the dimension of the space, and the order of derivatives is limited by the order of forming curves of the geometric interpolant.

\section{Conclusion}

In conclusion, we would like to note the need for further research and development of the proposed approach to modeling geometric manifolds with specified differential characteristics, which can take its place in the number of innovative numerical methods for solving differential equations. The prospect of further research is to conduct further computational experiments to test the accuracy and speed of the proposed approach to modeling geometric manifolds with specified differential characteristics and its practical application to the numerical solution of partial differential equations. It is assumed, that the use of a geometric interpolant as an analog of a finite super element, will allow us to obtain a numerical solution of partial differential equations and their systems of the required accuracy in the shortest possible time, even taking into account the nonlinear formulation of the original modeling problem. 


\section{References}

1. Vereshaga V. M., Bezditnyi A. A.: Modeling of functional curves of speed and distance in bn calculus. Bulletin of Kherson national technical University. 3 (50), 562-566 (2014).

2. Bezditnyi A. A.: Functional distance curve by means of BN-calculus. Applied geometry and engineering graphics. 4(48), 158-161 (2013).

3. Naidysh A. V., Baluba I. G., Bezditnyi A. A., Davydenko I. P. : Functional speed curve by means of BN-calculus / Applied geometry and engineering graphics. 4(56), 10-16 (2013).

4. Cottrell J.A., Hughes T.J., Bazilevs Y.: Isogeometric Analysis: Toward Integration of CAD and FEA. John Wiley \& Sons, Ltd. Chichester (2009).

5. Vuong A.-V.: Adaptive Hierarchical Isogeometric Finite Element Methods. Springer Spektrum. Wiesbaden. (2012).

6. Zaitsev V. F.: Symmetries of differential equations. Formal operators. https://cyberleninka.ru/article/n/symmetrii-differentsialnyh-uravneniy-formalnye-operational, last accessed 2019/03/08.

7. Yakovenko G. N.: Differential equations with fundamental solutions: Sophus Li and others. Fizmatkniga (2006).

8. Konopatskiy E. V.: Solving differential equations by geometric modeling methods. Proceedings of the 28th International conference on computer graphics and machine vision "GraphiCon 2018". Tomsk, pp. 322-325 (2018).

9. Konopatskiy E. V.: Modeling of an approximating 16-point compartment of the response surface, applied to the solution of a non-uniform heat equation. Geometry and graphics. 7(2), 38-45 (2019).

10. Konopatskiy E. V., Shevchuk O. A.: Use of geometric interpolants for numerical solution of differential equations. Information technologies: materials of the 84th scientific and technical conference of faculty, research staff and postgraduates (with international participation). Minsk, pp. 194-196 (2020).

11. Konopatskiy E. V.: Geometric theory of multivariate interpolation. Automation and simulation in engineering and management. Bryansk, 1(07), 9-16 (2020).

12. Balyuba I. G., Konopatskiy E. V., Bumaga A. I.: Point calculus: educational and methodological guide. Makeyevka (2020).

13. Konopatskiy E. V.: Modeling of arcs of curves passing through pre-set points. Bulletin of computer and information technologies. No. 2, pp. 30-36 (2019).

14. Konopatskiy E.V., Bezditnyi A.A.: Geometric modeling of multifactor processes and phenomena by the multidimensional parabolic interpolation method. IoP conference series: Journal of Physics: Conf. Series 1441012063 (2020).

15. Konopatskiy E. V.: Approximation of geometric objects using arcs of curves passing through pre-set points. Information technologies. 1(25), 46-52 (2019).

16. Konopatskiy E. V.: Geometric meaning of the method of least squares. Bulletin of computer and information technologies. No. 9, 11-18 (2019).

17. Konopatskiy E. V., Rotkov S. I.: Approximation of geometric objects of multidimensional space using arcs of curves passing through pre-set points. Proceedings of the 29th International conference on computer graphics and machine vision "GraphiCon 2019". Bryansk, pp. 191-195 (2009).

18. Fichtenholz G. M.: Course of differential and integral calculus. Book on Demand, Moscow, No.1 (2013). 